\title{
A smartphone-based survey in mHealth to investigate the introduction of the artificial intelligence into cardiology
}

\author{
Daniele Giansanti ${ }^{1}$, Lisa Monoscalco ${ }^{2}$ \\ ${ }^{1}$ Centro TISP, ISS, Roma, Italy; ${ }^{2}$ Facoltà di Ingegneria, Università di Tor Vergata, Roma, Italy \\ Contributions: (I) Conception and design: All authors; (II) Administrative support: None; (III) Provision of study materials or patients: All authors; \\ (IV) Collection and assembly of data: All authors; (V) Data analysis and interpretation: D Giansanti; (VI) Manuscript writing: All authors; (VII) Final \\ approval of manuscript: All authors. \\ Correspondence to: Daniele Giansanti. Centro TISP, ISS, Roma, Italy. Email: Daniele.giansanti@iss.it; gianslele@gmail.com.
}

\begin{abstract}
Background: There is an increasing discussion concerning the integration of artificial intelligence (AI) into medical decision-making. AI science is a branch of engineering that implements novel concepts to resolve complex challenges and defined as the theory and development of computer systems to perform tasks which would normally require human intelligence. AI could aid cardiologists in improving decision-making, workflow, productivity, cost-effectiveness, and ultimately, patient outcomes. The present study proposes a tool for a positioning exercise in cardiology using mobile technology.

Methods: This study is based on a dedicated tool with electronic surveys that collect the opinions, requirements, and desires of the interested actors including both laypeople and professionals.

Results: The tool was tested on 30 cardiologists and 30 subjects not involved in health care. The dataanalysis revealed several clear trends on the cardiologists: (I) a high desire to invest in AI; (II) high confidence in the use of $\mathrm{AI}$ in several fields of cardiology from risk prevention to diagnostics in medical imaging; (III) low confidence in the use of AI in quality control procedures; (IV) a strong belief that ethical issues are hampering the diffusion of AI to different fields. The data-analysis on the 30 subjects not involved in health care highlighted that $\mathrm{AI}$ is still not well known and therefore looked with suspicious.

Conclusions: The integration of AI with telemedicine and e-health is a key issue for the health care. The study highlights how the mobile technology-based positioning exercises in mHealth can be useful for health care decision makers.
\end{abstract}

Keywords: Survey; artificial intelligence; cardiology; mHealth

Received: 25 September 2019; Accepted: 17 June 2020; Published: 20 January 2021.

doi: $10.21037 /$ mhealth-19-188

View this article at: http://dx.doi.org/10.21037/mhealth-19-188

\section{Introduction}

"The medical decision" has always occupied a critical and central point in debate and discussions of modern medicine. In clinical practice, decision-making is a complex process that ideally is based on the availability of objective and reliable evidence, on immediate access to knowledge, on the correct interpretation $(1,2)$ of the facts available, and with integrating the risk-benefit relationship of the patient throughout this process (2). However (3), errors of judgment and decision-making inevitably occur and have been attributed mainly to two phenomena.

\section{Evaluation bias}

This is induced by a prejudice of the subject who perceives. Although no one is immune to cognitive distortion or cognitive bias, being aware of these phenomena can help; a generic component of cognitive distortion is present in any judgment, as it is linked to a perceptual factor and therefore to a vision of reality that is subjectively filtered by those who evaluate. 


\section{Noise in buman decision}

This is a well-known phenomenon that depends on human error factors that can affect the diagnostic chain. Decisions can also be influenced by apparently irrelevant factors attributable to those making the diagnosis, ranging from the current mood, to the time since the last meal, and the time dedicated to diagnosis.

For this reason, scholars and clinicians have been looking for a deus ex machina for decades that can give support and confidence to medical decision-making, and many have equated the arrival of this deus ex machina with the emergence of AI.

It seems that the discussion concerning the introduction of artificial intelligence (AI) into medical decision-making grows more intense each day and scholars are spiritedly (116) researching the application of $\mathrm{AI}$ in an array of medical processes, including risk-prevention and medical imagingbased diagnosis.

The use of AI in cardiology aims at improving decisionmaking, workflow, productivity, cost-effectiveness, and ultimately patient outcomes $(1,2,4)$. Thus far, AI has been used in automated predictions of cardiovascular disease risk scores and heart failure diagnosis. For instance, AI demonstrated its value by predicting heart failure 9 months before physicians who used traditional diagnostics could $(7,11,12)$. It has also proven its usefulness in analyzing data in multiple dimensions, and in one study, it was able to interpret echocardiograms using properly designed algorithms (8). Other authors have examined the application of $\mathrm{AI}$ in multiple-dimension imaging for diagnosis, including in determining the appropriateness of percutaneous coronary intervention and treatment (13). Naturally, a precise prediction on the development of AI in cardiology is not yet possible. Much depends on how we will deal with the impact that AI will have on bioethical aspects and on the opinion of subjects involved or not in health care and, very important, how stakeholders respond to these issues. However, although it is not possible to give a specific assessment of this new opportunity, identifying those areas that will benefit from AI is still feasible. Johnson et al. (10) reported, for example, the possible development of $\mathrm{AI}$ in cardiology comparable to the active role $\mathrm{AI}$ may have in research and development, clinical practice, and the direct relationship with the health of the population. An important role in designing this revolutionary scenario will be played by the actors themselves who work in these areas, from traditional to interventional cardiology. Precisely for this reason, it will be important to conduct dedicated positioning exercises, in particular for those working in the front line, using, using, for example, dedicated surveys implemented through modern technology.

The debate on the use of AI in cardiology is now underway. Recent studies reported increasing interest on AI. However, it is still important to consider opinions of all those involved in the work chain through specific feedback. It is therefore necessary to design a methodology that, by means of a wide interaction with the involved actors, can facilitate the investigation for the introduction of AI in cardiology. In order to reach this objective, we decided to develop electronic tools compatible with a mobile technology to perform dedicated surveys. Using this approach, we could obtain tangible and quantifiable information in (I) a collaborative and (II) automated manner. This information could be useful for the stakeholders and therefore could be useful to enable decisions in this field.

\section{Methods}

\section{Development of survey tools}

\section{Traditional paper-based surveys}

The survey undoubtedly represents a powerful tool for investigating many scientific problems, and, as such, is a valuable, if not fundamental apparatus for addressing the issues mentioned in this study, in which remotely gathering information/opinion from the actors involved in this field is essential. A problem that immediately emerges is that of administration and data collection, given that in complex administrations and/or when large numbers are managed, the management of the process becomes laborious and complicated. In particular, when using paper-based tools, the following difficulties arise: complexity in reaching all the actors, complexity in collecting paper feedback, difficulty and tediousness in manually inserting the data from paper into appropriate databases for analysis (such as Excel) with the possibility of error.

\section{Electronic-based surveys}

To overcome these difficulties, electronic surveys (eSs) can be used to provide the following advantages:

(I) Easy administration: it is possible to send an Internet link through the most common web communication tools (e-mail, messenger).

(II) Automatic data storing in the cloud: the recipients, once the link has been selected, can access the 


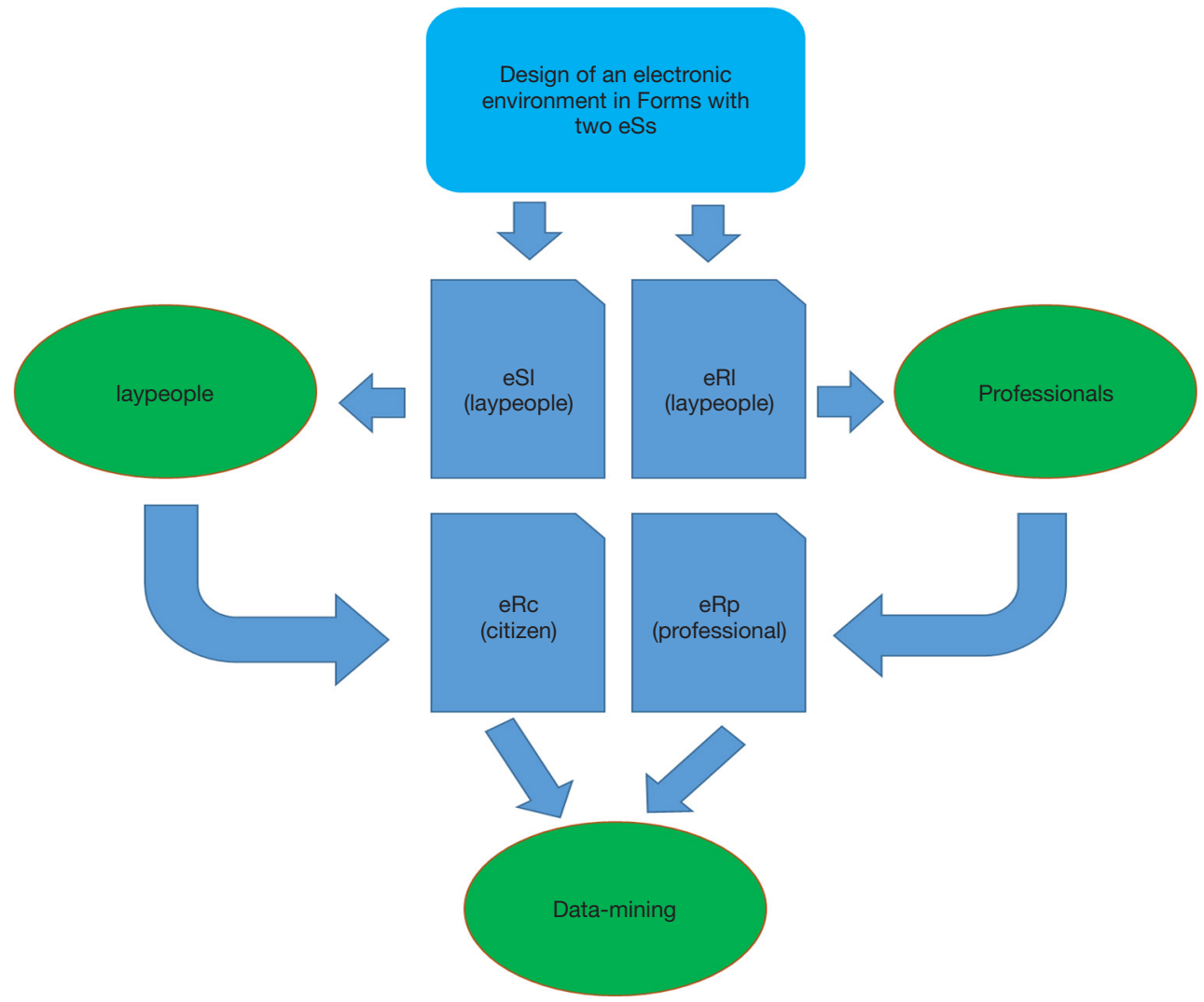

Figure 1 The methodological flow.

survey online, fill it in, and complete it by means of an automatic entry of data into the appropriate databases (without manual operation). Once the interested parties complete the questionnaire, the data entered are automatically loaded into a database.

The giants of computer science, like Microsoft and Google, have made several electronic survey applications available.

\section{Designing the electronic survey}

Microsoft, for example, provides their users (who have a Microsoft account) two solutions eS solutions; both of these solutions allow the remote distribution of an eS to the desired actors by simply sending an Internet link and collecting the data in a cloud. The first one, OneDrive, stores the data in the cloud in the Excel Online format; the second one, Forms, arranges the data in the cloud into useful Excel format reports (self-updated each time an online eS is completed); the report can be shared by simply providing an internet link. After a comparison study, we chose Forms (17) and designed two eSs for the actors; the first was an eS for laypeople (eSl), the second was an eS for the professionals specializing in this field (eSp).

Figure 1 highlights the proposed flow, from the design of the eSs to the data mining obtained on the basis of the two automatically updated electronic reports (eRs) for the laypeople (eRl) and for the professionals (eRp).

\section{Results}

\section{The survey}

The first result is represented by the environment with the four core elements (eSl, eSp, eRl, eRp).

Figure $2 A, B$ show the two Quick Response codes related to the two surveys eSc and the eSp (obviously related to a mirror version of the original). Figure 3 shows the print screens related to the survey proposed for the professionals.

At the moment, we have submitted the survey to 30 professionals and 30 laypeople. Table 1 shows the two 
groups; all the laypeople (lp) had a high secondary school level of education. Table 2 shows the values related to the self-assessment of the preliminary knowledge on the AI and the opinion on the introduction of AI. All the values related

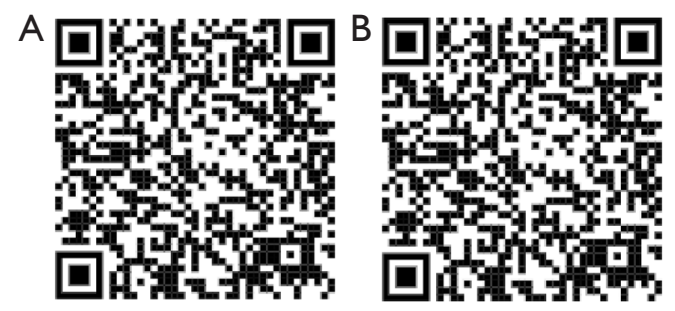

Figure 2 The quick response codes of the two surveys for the laypeople (A) and for the professionals (B). to the knowledge (from \#1 to \#5) were over the threshold $[3,50]$ for the cardiologists. The highest value was related to knowledges of technologies for biomedical applications in cardiology; this value was evidently influenced by educational path. Table 2 also highlights that for the cardiologists the opinions on the introduction of AI both in general medicine and in cardiology received an assessment over the threshold (\#6 and \#7), while the "introduction of AI in quality control procedures" received an assessment under the threshold (\#8) indicating a low degree of confidence for AI in quality control. The same Table 2 shows that for the $1 p$ (from \#9 to \#16) the same investigated aspects received all a value always under the threshold and never higher than 2.3.

Table 3 highlights, with the reference to the cardiologists, the assessment on the use of AI in several specific
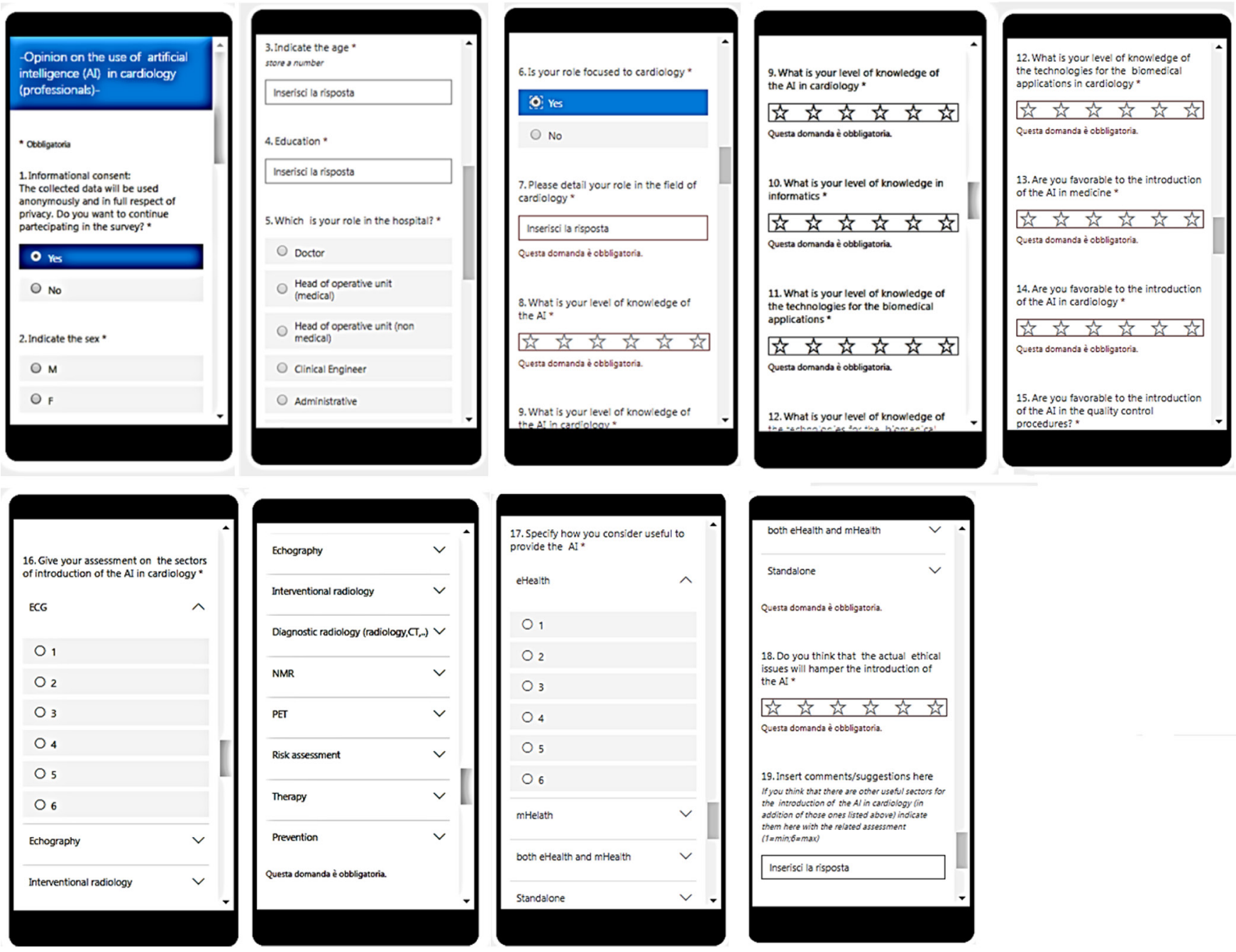

Figure 3 Print screens of the survey related to the professionals. 
Table 1 Subjects involved in the study

\begin{tabular}{lccc}
\hline Group & Sex & Mean age (y) & Standard deviation (y) \\
\hline Cardiologists & Males (50\%) & 47.3 & 2.5 \\
Cardiologists & Females (50\%) & 48.1 & 2.7 \\
Laypeople & Males (50\%) & 46.2 & 6.6 \\
Laypeople & Females (50\%) & 45.1 & 6.9 \\
\hline
\end{tabular}

Table 2 Preliminary knowledge on the introduction of the AI and opinions on the introduction of the AI for the two investigated groups

\begin{tabular}{|c|c|c|c|c|}
\hline \# & Group & Question on & Mean & Standard deviation \\
\hline 1 & Cardiologists & Knowledge on "Al" & 4 & 0.3 \\
\hline 2 & Cardiologists & Knowledge on "Al in cardiology" & 4.2 & 0.2 \\
\hline 3 & Cardiologists & Knowledge on "Al in informatics" & 4.1 & 0.3 \\
\hline 4 & Cardiologists & Knowledge on "Tech for biomed apps" & 4.2 & 0.3 \\
\hline 5 & Cardiologists & Knowledge on "Tech for biomed apps in cardiology" & 4.8 & 0.3 \\
\hline 6 & Cardiologists & Opinion on the "Use of $\mathrm{Al}$ (in general)" & 4.5 & 0.3 \\
\hline 7 & Cardiologists & Opinion on the "Use the Al in cardiology" & 4.5 & 0.2 \\
\hline 8 & Cardiologists & Opinion on the "Use of Al in quality control procedures" & 3.4 & 0.3 \\
\hline 9 & Laypeople & Knowledge on "Al" & 1.8 & 0.3 \\
\hline 10 & Laypeople & Knowledge on "Al in cardiology" & 2.2 & 0.2 \\
\hline 11 & Laypeople & Knowledge on "Al in informatics" & 2.1 & 0.3 \\
\hline 12 & Laypeople & Knowledge on "Tech for biomed apps" & 2.2 & 0.3 \\
\hline 13 & Laypeople & Knowledge on "Tech for biomed apps in cardiology" & 2.3 & 0.3 \\
\hline 14 & Laypeople & Opinion on the "Use of Al (in general)" & 1.9 & 0.2 \\
\hline 15 & Laypeople & Opinion on the "Use the Al in cardiology" & 1.9 & 0.2 \\
\hline 16 & Laypeople & Opinion on the "Use of Al in quality control procedures" & 1.8 & 0.2 \\
\hline
\end{tabular}

$\mathrm{Al}$, artificial intelligence.

fields of cardiology (from \#1 to \#9) and on the mode of implementation (from \#10 to \#13). Three trends are worth noting. First, all the indicated fields received an assessment higher than the threshold. Second, medical imaging received the lowest confidence. Third, electrocardiography, risk assessment, prevention, and therapy received the highest degree of confidence. In terms of the mode of AI implementation, the standalone model was preferred as compared to the eHealth and mHealth approaches, which, as is well known, may recur to the connection to the networks and therefore may be exposed to the cyber-risks. Indeed, cardiologists widely acknowledge that networks entail risks related to cyber security, and this surely affected the latter result. It is thus clear that ethical issues will hamper the introduction of $\mathrm{AI}$ (mean 5.2; standard deviation 0.2).

The same aspects investigated on the $1 p$ showed a value always lower than the threshold and lower than 1.7 in mean value. Furthermore, this value was lower than the one related to the first section of the investigation (see Table 2) dedicated to this group. This indicates that when going into details on the medical applications the assessment of the investigated parameters further decreases. 
Table 3 Assessment on the introduction of AI in several fields of cardiology and on mode of implementation (using eHealth, mHealth, and a standalone approach): results for the cardiologists

\begin{tabular}{llcc}
\hline$\#$ & Opinion on the integration into & Mean & Standard deviation \\
\hline 1 & Electrocardiography & 4.7 & 0.3 \\
2 & Echography & 3.8 & 0.3 \\
3 & Interventional radiology & 3.9 & 0.2 \\
4 & Diagnostic radiology (radiology. CT...) & 3.9 & 0.3 \\
5 & Nuclear magnetic resonance & 3.8 & 0.2 \\
6 & Positron emission tompgraphy & 3.8 & 0.3 \\
7 & Risk assessment & 5.2 & 0.2 \\
8 & Therapy & 5.1 & 0.2 \\
9 & Prevention & 5.1 & 0.2 \\
10 & Mobile health & 4.1 & 0.3 \\
11 & Electronic health & 4.3 & 0.2 \\
12 & Both electronic health and mobile health & 4.2 & 0.3 \\
13 & Standalone & 5.2 & 0.3 \\
\hline
\end{tabular}

$\mathrm{Al}$, artificial intelligence.

\section{Discussion and conclusions}

The present study considers the integration of AI with cardiology $(1-15,17,18)$ and proposes a methodology that, by means of a wide interaction of the involved actors, allows a positioning exercise for the use of AI in cardiology. The methodology was based on a properly designed environment based on Microsoft Forms that allowed a detailed investigation, data collection, and analysis starting from the submission of electronic surveys to professionals and laypeople involved in this field up to the first data mining from the electronic reports. The first added value of the study is represented by the solution based on electronic surveys. The second added value is the availability of a specific solution for investigating the introduction of AI in cardiology. The final added values are the outputs from the electronic surveys submitted both to laypeople and professionals. They could be useful for stakeholders in the field to address studies and propose initiatives.

The data analysis from the cardiologists yielded several noteworthy observations:

- A high level of self-assessed basic knowledge of AI, informatics, and medical technologies;

- A high general desire for the introduction of AI to various fields of the cardiology;

- A higher preference for the use of AI in applications not related to medical imaging;

- A low level of confidence on the use of AI in quality control procedures;

- A preference for the use of standalone AI compared to using AI with networks (eHealth; mHealth);

- An openness to future expansion of the study on the basis of stakeholder initiatives.

The data analysis from the laypeople yielded two important considerations. The first consideration is that, although the subjects have an average culture, they have a very low knowledge of AI, both in general and in the medical field. This awareness decreases when one enters in more detail in the medical aspects. The second consideration is that the introduction of AI in cardiology cannot overlook the views of these citizens who currently view the AI with suspicion.

The results from the cardiologists reflects their opinion that AI could represent a solution to overcome the limits represented by the traditional non-AI technologies [their hope is that AI (1-3) can circumvent the common causes of incorrect diagnoses: evaluation bias and noise]; while the results from laypeople, future health clients of AI solution, shows diffidence on AI. Many studies have been done in the context of the introduction of AI cardiology. There is no doubt that 
these studies have shown the potential of $\mathrm{AI}$ in different areas of cardiology (9) and in different applications ranging from risk analysis to imaging (7,8,11-13). However, now it is strongly needed, to start thinking about the last mile of the introduction of AI in cardiology. In our study, we have made a contribution in this direction. In fact, the last mile passes through the opinions of the actors in this area which include both the health service operators and citizens, in other words on the crucial aspect of the acceptance of AI (2). Our surveys have begun to gather these fundamental opinions through automatic (17) and easily administered on a large scale methodologies. This is a very important step in introducing $\mathrm{AI}$ into eHealth and mHealth. Furthermore, these methodologies and results could be used, for example, in specific bealth technology assessment studies $(19,20)$ as happened in other sectors $(21)$ after appropriate remodeling for this specific sector.

\section{Appendix: the link to the two surveys}

The links for the eSl and the eSp (obviously related to a mirror version of the original reserved for experimentation) are provided below:

https://forms.office.com/Pages/ResponsePage.aspx?id=D QSIkWdsW0yxEjajBLZtrQAAAAAAAAAAAAZ_.gdk7kp UNEhTUFVLV11PQ0JDSDNZTEIXNkNDUUtMUy4u

https://forms.office.com/Pages/ResponsePage.aspx?id=D QSIkWdsW0yxEjajBLZtrQAAAAAAAAAAAAZ__gdk7kp URFJSVFU3U0dXRUtJOEJNNTJOTU4zUTZRRS4u

\section{Acknowledgments}

Funding: None.

\section{Footnote}

Provenance and Peer Review: This article was commissioned by the Guest Editors (Sheikh Mohammed Shariful Islam and Ralph Maddison) for the series "Digital Health for Cardiovascular Disease" published in the mHealth. The article was sent for external peer review organized by the Guest Editors and the editorial office.

Conflicts of Interest: All authors have completed the ICMJE uniform disclosure form (available at http://dx.doi. org/10.21037/mhealth-19-188). The series "Digital Health for Cardiovascular Disease" was commissioned by the editorial office without any funding or sponsorship. DG serves as an unpaid editorial board member of the mHealth from Apr 2019 to Apr 2021. The authors have no other conflicts of interest to declare.

Ethical Statement: The authors are accountable for all aspects of the work in ensuring that questions related to the accuracy or integrity of any part of the work are appropriately investigated and resolved. The study did not directly involve any human subjects in clinical trials. Subjects (all healthy) were involved anonymously in a survey. The ethical approval was not judged necessary. The consent forms were not therefore applicable. At the starting of the survey we gave the information about the anonymous data insertion/management.

Open Access Statement: This is an Open Access article distributed in accordance with the Creative Commons Attribution-NonCommercial-NoDerivs 4.0 International License (CC BY-NC-ND 4.0), which permits the noncommercial replication and distribution of the article with the strict proviso that no changes or edits are made and the original work is properly cited (including links to both the formal publication through the relevant DOI and the license). See: https://creativecommons.org/licenses/by-nc-nd/4.0/.

\section{References}

1. Miyazawa AA. Artificial intelligence: the future for cardiology Heart 2019;105:1214.

2. Bonderman D. Artificial intelligence in cardiology. Wien Klin Wochenschr 2017;129:866-8.

3. Kahneman D, Rosenfield AM, Gandhi L, et al. How to overcome the high, hidden cost of inconsistent decision making. Harv Bus Rev 2016;10:36-43.

4. Stevenson A. Oxford dictionary of English. 3rd edn. Oxford: Oxford University Press, 2010.

5. Constantinides P, Fitzmaurice DA. Artificial intelligence in cardiology: applications, benefits and challenges. Br J Cardiol 2018;25:1-3.

6. Tajik AJ. Machine Learning for Echocardiographic imaging: embarking on another incredible journey. J Am Coll Cardiol 2016;68:2296-8.

7. Krittanawong C, Zhang H, Wang Z, et al. Artificial intelligence in precision cardiovascular medicine. J Am Coll Cardiol 2017;69:2657-64.

8. Zhang J, Gajjala S, Agrawal P, et al. Fully automated echocardiogram interpretation in clinical practice. Circulation 2018;138:1623-35. 
9. Mullainathan S, Obermeyer Z. Does machine learning automate moral hazard and error? Am Econ Rev 2017;107:476-80.

10. Johnson KW, Torres Soto J, Glicksberg BS, et al. Artificial intelligence in cardiology. J Am Coll Cardiol 2018;71:2668-79.

11. Shah SJ, Katz DH, Selvaraj S, et al. Phenomapping for novel classification of heart failure with preserved ejection fraction. Circulation 2015;131:269-79.

12. Choi E, Schuetz A, Stewart WF, et al. Using recurrent neural network models for early detection of heart failure onset. J Am Med Inform Assoc 2017;24:361-70.

13. Davies J. CEREBRIA-1: machine learning vs expert human opinion to determine physiologically optimized coronary revascularization strategies. Presented at: TCT 2018. September 24, 2018. San Diego, CA.

14. Swaine E. Healio. Machine-learning algorithm improves iFR measurement quality. Available online: https://www. healio.com/cardiac-vascular-intervention/percutaneouscoronary-intervention/news/online/\% 7B759e6b14462a-4bb3-95dd-5f8739b3822f\% 7D/machine-learningalgorithm-improves-ifrmeasurement-quality (Accessed: 05 Nov 2018).

15. Maxwell Y. TCTMD. Human vs machine: computer

doi: $10.21037 /$ mhealth-19-188

Cite this article as: Giansanti D, Monoscalco L. A smartphone-based survey in mHealth to investigate the introduction of the artificial intelligence into cardiology. mHealth 2021;7:8. algorithm matches experts for PCI appropriateness and planning. 2018. Available online: https://www.tctmd. com/news/humanvs-machine-computer-algorithmmatches-experts-pciappropriateness-and-planning (Accessed 22nd Oct 2018).

16. Dawes TJW, de Marvao A, Shi W, et al. Machine learning of three-dimensional right ventricular motion enables outcome prediction in pulmonary hypertension: a cardiac MR imaging study. Radiology 2017;283:381-90.

17. Giansanti D, Grigioni M. Chapter 9, Istisan Report 18/21, ISS, Roma Italy.

18. Lau E, Watson KE, Ping P. Connecting the dots: from big data to healthy heart. Circulation 2016;134:362-4.

19. Giansanti D, Morelli S, Macellari V. Telemedicine technology assessment part II: tools for a quality control system. Telemed J E Health 2007;13:130-40.

20. Giansanti D, Morelli S, Macellari V. Telemedicine technology assessment part I: setup and validation of a quality control system. Telemed J E Health 2007;13:118-29.

21. Giansanti D, Pochini M, Giovagnoli MR. Integration of tablet technologies in the e-laboratory of cytology: a health technology assessment. Telemed J E Health 2014;20:909-15. 